\title{
Pulsar polarization: the view from the southern hemisphere
}

\author{
Simon Johnston \\ CSIRO Astronomy and Space Science, \\ PO Box 76, Epping, NSW 1710, Australia. \\ email: simon. johnston@csiro.au
}

\begin{abstract}
Pulsar polarization has been a fruitful area of study since the first discovery of pulsars 50 years ago. Polarization gives information on the geometry of the star, the location of the radio emission in the magnetosphere, the physics behind the radio emission mechanism and a plethora of phenomenology. Here, I will restrict myself to a brief outline of recent work in pulsar polarization using observations taken with the Parkes radio telescope over the past decade.
\end{abstract}

Keywords. pulsars:general

Almost immediately after the discovery of pulsars it was realised that the emission from these stars is highly linearly (Lyne \& Smith 1968) and circularly polarized (Craft et al. 1968). Indeed the degree and complexity of the radio polarization in both single pulses and integrated profiles became one of the defining characteristics of pulsars.

In this short paper, it is not possible to do justice to 50 years of advances in pulsar polarization observation and theory. Important early papers in the field which serve as an introduction for the general reader include the development of the rotating vector model for polariation by Radhakrishnan \& Cooke (1969), which in turn led to the hollowcone idea for the beam pattern of pulsars (Komesaroff 1970). Seminal papers from the 1980s are the beam models of Rankin (1983) and Lyne \& Manchester (1988), with the former advocating a structured cone-core paradigm whereas the latter favour a more random, patchy model of the beam structure. Finally, Blaskiewicz et al. (1991) included relativistic effects and showed how to determine the emission heights based on the delay of the total intensity profile relative to the maximum swing of the position angle of the linear polarization.

Here I will limit myself to presenting four main results based on polarization observations with the Parkes telescope over the last decade and lay out some the puzzles which arise from these observations.

\section{Magnetars}

There are four radio magnetars now well studied in polarization; PSRs J1809-1943 (Camilo et al. 2007, Kramer et al. 2007), J1550-5418 (Camilo et al. 2008), J1622-4950 (Levin et al. 2012) and the Galactic Centre magnetar (Torne et al. 2017). There are strong similarities between the four objects. They all have flat spectral index with emission visible to high frequencies, they have a high degree of linear polarization, the profiles are wide and vary with time and the flux density varies from off to extremely bright (see Figure 1, top left panel). Analysis of the position angle sweep of the linear polarization seems to imply that the inclination angles are small. Key questions which arise from the polarization observations are then (a) why does the rotating vector model fit the data 

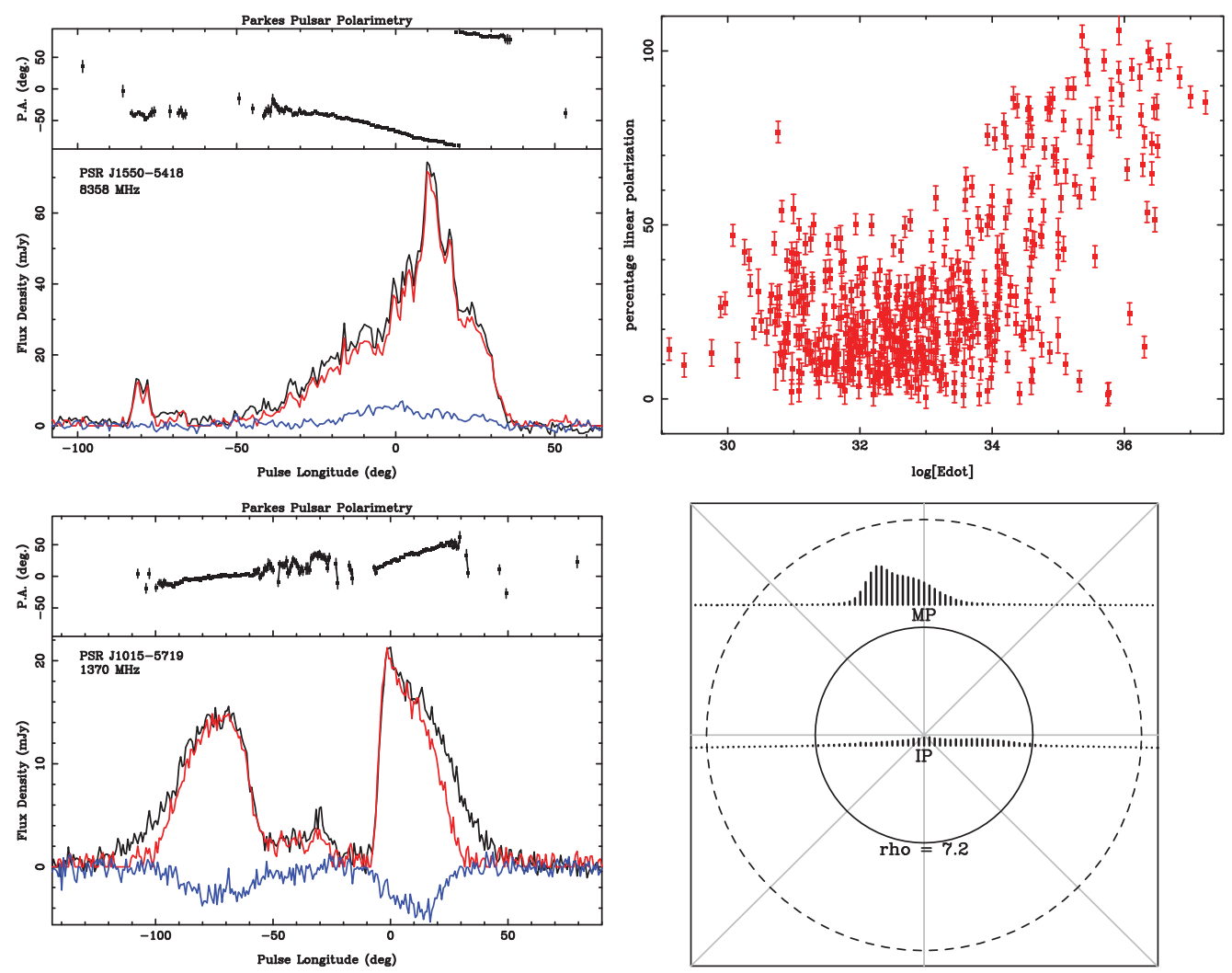

Figure 1. Top left: Polarization of the radio magnetar PSR J1550-5418 taken from Camilo et al. (2008). Total intensity, linear polarization, and circular polarization are shown in the bottom panel and the position angle of the linear polarization in the top panel. Top right: Percentage linear polarization versus $\dot{E}$ for 500 pulsars taken from Johnston \& Kerr (2017). Bottom left: PSR J1015-5719, an example of a typical high $\dot{E}$ pulsar profile taken from Johnston \& Weisberg (2006). Bottom right: The beam map for the interpulse pulsar PSR J0627+0706 taken from Keith et al. (2010).

so well, when the magnetic fields of magnetars are expected to be very non-dipolar? (b) is the alignment of the rotation and magnetic axes a feature (or cause) of (radio) magnetars?

\section{Alignment of velocity and rotation axes}

Theoretical work by Spruit \& Phinney (1998) showed that the velocity axis and the rotation axis of pulsars should be aligned during the birth process. Observational evidence in the form of X-ray torii also seemed to point in this direction (Helfand et al. 2001, Romani \& Ng 2003, Romani et al. 2005). We therefore undertook a campaign to use radio polarization information to determine the rotation axis of a sample of young pulsars. This procedure requires absolute polarization calibration (van Straten et al. 2010) and a determination of the position angle of the linear polarization at the inflexion point (magnetic axis) of the RVM curve. Johnston et al. (2005) determined that indeed the velocity and rotation vectors align, furthermore some pulsars appear to emit predominantly in the X-mode and some in the O-mode. Follow-up observational papers using an expanded dataset include Johnston et al. (2007), Rankin (2007) and Noutsos et al. 
(2012). In a summary paper which included results for 58 pulsars, Noutsos et al. (2013) considered the implications on the spin periods of pulsars at birth and on the various mechanisms for supernova kicks. From a pulsar emission physics point of view, how is it that some pulsars emit only in X-mode and some only in O-mode; perhaps refraction plays an important role.

\section{High $\dot{E}$ and low $\dot{E}$ pulsars}

There is a distinct difference between high $\dot{E}$ and low $\dot{E}$ in both total intensity and polarization. Many high $\dot{E}$ pulsars have wide profiles with a double-pulse structure with a dominant second (trailing) component and increased circular polarization also in the trailing component (Johnston \& Weisberg 2006; see Figure 1 bottom left panel). The PA sweep and use of the Blaskiewicz et al. (1991) formalism shows that the emission height is relatively high in these high $\dot{E}$ pulsars (Karastergiou \& Johnston 2007). In general, the linear polarization is very high, often approaching $100 \%$ (Weltevrede \& Johnston 2008 ), unlike the low $\dot{E}$ pulsars which usually have fractional polarization less than $30 \%$ (Johnston \& Kerr 2017). The differences between the two sets of pulsars appears to set in at $\dot{E} \sim 10^{34.5} \mathrm{erg} / \mathrm{s}$ (see Figure 1 top right panel).

\section{Emission from outside the polar caps}

Generally, it is believed that the radio radiation flows out along the open magnetic field lines; the locus of these field lines forms the polar cap. Recent evidence, however, suggests that some radio emission might be seen from outside this conventional polar cap region. Weltevrede \& Wright (2008) considered the geometry of the interpulse pulsar PSR J1057-5226 (B1055-52) and concluded that the main pulse emission must originate from fieldlines from well outside the polar cap boundary beyond the null surface. Prompted by this result, Keith et al. (2010) examined other interpulse pulsars (see Figure 1 bottom right panel) and came to a similar conclusion, at least in come cases. Rookyard et al. (2015b) examined the geometry of a large sample of $\gamma$-ray pulsars where the inclination between the magnetic and rotation axes is expected to be large. Surprisingly, however, low values were measured. This led Rookyard et al. (2015a) to surmise that the solutiuon to this problem lay in the assumption that emission only came from the polar cap region. Relaxing this assumption helps to fix the problem (see Weltevrede's paper in these proceedings). This work raises all sorts of questions including (a) the reliability of angles derived from RVM, (b) the applicability of retardation/aberration, (c) the filling factor of pulsar beams, (d) gamma-ray emission models.

\section{Future Work}

An upcoming publication will provide a public archive of 600 pulsar profile in full polarization and associated statistics from Parkes observations over the past decade. For details of how to access the data please see Johnston \& Kerr (2017). The Parkes telescope will be upgraded shortly with a wide-band receiver capable of observing from $700-4000$ $\mathrm{MHz}$ simultaneously; this promises to be an exciting new development for polarization. The MeerKAT telescope is coming on-line, its improved sensitivity compared to Parkes will make it a key instrument for southern hemisphere pulsars. Finally, the SKA promises to solve all problems (!!) and an outline of possible pulsar polarization science is given in Karastergiou et al. (2015). 


\section{Acknowledgments}

I thank the organisers for putting on a fabulous meeting. Many thanks to all my collaborators on pulsar polarization topics, it's been a lot of fun. Last but not least, kudos to the "Rainbow Unicorn" team for blitzing the trivia quiz. Champions!

\section{References}

Blaskiewicz, M., Cordes, J., \& Wasserman, I. 1991. ApJ, 370, 643

Camilo, F., Reynolds, J., Johnston, S., Halpern, J., Ransom, S., \& van Straten, W. 2007. ApJ, 659, L37

Camilo, F., Reynolds, J., Johnston, S., Halpern, J., \& Ransom, S. 2008. ApJ, 679, 681

Craft, H., Comella, J., \& Drake, F. 1968. Nature, 218, 1122

Helfand, D., Gotthelf, E., \& Halpern, J. 2001. ApJ, 556, 380

Johnston, S., Hobbs, G., Vigeland, S., Kramer, M., Weisberg, J., \& Lyne, A. 2005. MNRAS, 364,1397

Johnston, S., Kramer, M., Karastergiou, A., Hobbs, G., Ord, S., \& Wallman, J. 2007. MNRAS, 381,1625

Johnston, S., \& Kerr, M. 2017. MNRAS, In Press

Johnston, S. \& Weisberg, J. 2006. MNRAS, 368, 1856

Karstergiou, A. et al. 2015. Proceedings of Science, AASKA14.

Karastergiou, A. \& Johnston, S. 2007. MNRAS, 380, 1678

Keith, M., Johnston, S., Weltevrede, P., \& Kramer, M. 2010. MNRAS, 402, 745

Komesaroff, M. 1970. Nature, 225, 612

Kramer, M., Stappers, B., Jessner, A., Lyne, A., \& Jordan, C. 2007. MNRAS, 377, 107

Levin, L. et al. 2012. MNRAS, 422, 2489

Lyne, A. \& Manchester, R. 1988. MNRAS, 234, 477

Lyne, A. \& Smith, F. 1968. Nature, 218, 124

Noutsos, A., Kramer, M., Carr, P., \& Johnston, S. 2012. MNRAS, 423, 2736

Noutsos, A., Schnitzeler, D., Keane, E., Kramer, M., \& Johnston, S. 2013. MNRAS, 430, 2281

Radhakrishnan, V. \& Cooke, D. 1969. Ap. L., 3, 225

Rankin, J. 1983. ApJ, 274, 333

Rankin, J. 2007. ApJ, 664, 443

Romani, R. \& Ng, C-Y. 2003. ApJ, 585, L41

Romani, R., Ng, C-Y., Dodson, R., \& Brisken, W. 2005. ApJ, 631, 480

Rookyard, S., Weltevrede, P., \& Johnston, S. 2015a. MNRAS, 446, 3356

Rookyard, S., Weltevrede, P., \& Johnston, S. 2015b. MNRAS, 446, 3367

Spruit, H. \& Phinney, E. 1998. Nature, 393, 139

Torne, P. et al. 2017. MNRAS, 465, 242

van Straten, W., Manchester, R., Johnston, S., \& Reynolds, J. 2010. PASA, 27, 104

Weltevrede, P. \& Johnston, S. 2008. MNRAS, 391, 1210

Weltevrede, P. \& Wright, G. 2008. MNRAS, 395, 2117 